\title{
Providing Variable-Rate Transmission for the Integrated Multimedia Services in the CDMA Network
}

\author{
*KyungSu Park and ${ }^{* *}$ DongHo Cho \\ * Senior Research Engineer, R\&D Center, Mediacomm, Inc., KAIST Suite 7223, \\ 207-43, Cheongryangri-dong, Dongdaemun-gu, Seoul 130-012, Korea \\ Tel : +82-2-959-0334(\#107) / Fax : +82-2-969-0239 / E-Mail : kspark@infocomm.kyunghee.ac.kr \\ ** Associate Professor, Dept. of Electrical Eng., KAIST \\ 373-1, Kusong-dong, Yusong-gu, Taejon 305-701, Korea \\ Tel : +82-42-869-3467 / Fax : +82-42-869-3410 / E-Mail : dhcho@ee.kaist.ac.kr
}

\begin{abstract}
In this paper, we propose wireless medium access control protocol for CDMA network based on contention and reservation, which provides mobile terminals with connection-oriented/connectionless services and supports various bit rates required to support integrated multimedia services. Proposed mechanism improves utilization efficiency of spreading codes by allowing limited spreading codes to be shared among all mobile terminals. Mobile terminals can transmit user traffic using multiple spreading codes. Also, depending on the number of usable uplink slots within frame and the number of spreading codes usable within each slot, various bit rates can be provided for each mobile terminal. Proposed mechanism allows connectionoriented and connectionless services, and optimum bit rates are supported for each service. Also, we analyze the performance of proposed wireless MAC protocol in view of mean blocking rate of connection-oriented services and mean delay time of connectionless services.
\end{abstract}

\section{Introduction}

Mobile communication networks based on CDMA, which provides most efficient frequency reusability among neighboring cells, have been designed and developed mainly for voice services[1]. However, in recent, there are increasing numbers of users requiring various multimedia services including data, fax and video traffics. Especially, there are many researches on the wircless ATM, which extends ATM transmission mechanism to the area between access point and mobile terminal[5]-[7]. To provide multimedia services through wireless channels, it is important to satisfy required QoS of each service. It can be accomplished by employing many schemes, one of which is priority control and optimum resource allocation mechanism according to the traffics classes with different QoS requirements in view of maximum allowable delay, mean/peak transmission rate, error control and other factors[6]. Also, most efficient usage of wireless channel has to be accomplished because its capacity is very limited compared with wired networks.

To satisfy maximum allowable delay requirements of various traffics, mobile terminals must use connectionoriented service with dedicated channels, or connectionless service competing shared channels with different priorities among all terminals. To satisfy error control requirements of various traffics, we can apply different error control mechanisms according to traffic types. Meanwhile, to transmit multimedia traffics with various mean/peak bit rates according to applications, connection-oriented and connectionless mobile terminals have to be able to use variable capacity of uplink channels if necessary. Thus, wireless MAC protocol layer of each mobile terminal should provide proper mechanisms to request and use variable channel capacity, and should support connection-oriented and connectionless services to upper layers.

In the CDMA network with a given channel bandwidth or a maximum acceptable time delay for code tracking and synchronization, the number of spreading codes is limited[1]. Thus, how to fully exploit the limited code resource to provide integrated multimedia services is very important. In conventional CDMA networks, each mobile terminal is served with its own spreading code. However, for integrated services including various multimedia traffics as well as voice, various bit rates transmission should be supported over the common air interface(CAI)[6]. Therefore, channel utilization efficiency has to be improved by allowing limited spreading codes shared among mobile terminals. This enables CDMA network to provide many kinds of multimedia services requiring various bit rates, and to support higher capacity based on given channel bandwidth. In this paper, we propose new wireless MAC protocol sharing spreading code, and analyze its performance in view of mean blocking rate of connectionoriented services and mean delay time of connectionless services.

\section{Spreading code sharing mechanism in CDMA network}

A. PCRMA channel access mechanism

The proposed CDMA wireless MAC protocol is named as packet code reservation multiple access(PCRMA). Since downlink channels are used by AP to transmit forward traffics based on internal scheduling, there are no collisions and QoS can be guaranteed in maximum. Therefore, we investigate into only uplink channel access mechanism. PCRMA is designed to provide multimedia services with various bit rates, and supports both the connection-oriented and connectionless services. Here, resource allocation is controlled by central AP, satisfying QoS requirements of each traffic type.

PCRMA uplink channel structure is shown in Fig.1.AP broadcasts the information of spreading codes available in next uplink slot, through downlink control channel. Based 
<Table.I> Status information messages of uplink spreading code

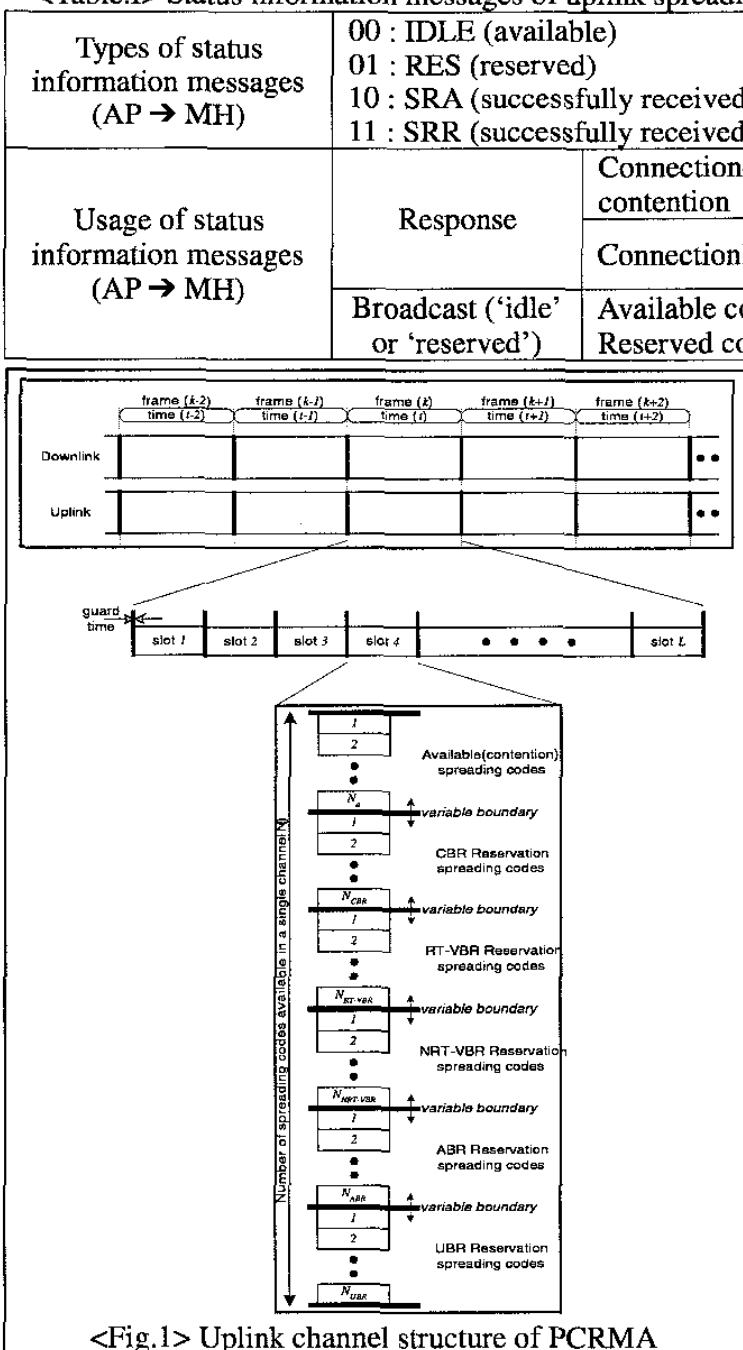

on this information, mobile terminals which have user traffics to transmit to AP access uplink channel using available spreading code in next slot. Mobile terminal requiring connection-oriented service transmits channel reservation requirement packet, which includes Qos information like required bit rate, maximum allowable delay and etc., at the first channel access time. When AP receives this packet, it decides whether there are enough uplink channel resources to support required QoS. If sufficient channel resources are available, AP transmits feedback message which includes one or more slot numbers and spreading codes reserved for the mobile terminal. Receiving this feedback message, mobile terminal transmits user traffic using allocated channel resources(slots and codes) at next uplink slot, and transmits user traffics using one or more allocated spreading codes in each reserved slot. However, if multiple terminals transmit channel request packet using the same available spreading code, there occurs collision. In this case, AP transmits no feedback message through downlink slot, and mobile terminals select one of available spreading codes randomly and retransmit channel request packet at next uplink slot.

Table I shows the broadcast messages related with uplink channel status information. These messages can be used as response to the channel request or packet data transmission from mobile terminals.

In PCRMA, each uplink frame is divided into multiple slots. Mobile terminals can transmit packets by using multiple codes in each slot. PCRMA network provides connection-oriented service and connectionless packet transmission service according to traffic type. Mobile terminals using connection-oriented service are assigned with proper slots and spreading codes can be used in each slot, according to required bit rate. However, in the case of connectionless service, mobile terminal transmits $j$ packets by using $j$ available spreading codes selected from a pool of all available codes in each slot. Therefore, in the case of connectionless service, collision can be occurred if same spreading code is used by more than two mobile terminals.

Thus, mobile terminals can be provided with various bit rates according to both the number of slots used to transmit packet as well as the number of spreading codes reserved in each slot. Also, in the respect of system, efficient usage of wireless channels can be obtained because exactly required amount of slots and spreading codes are assigned for each mobile terminal. There can be various combination of the number of slots and spreading codes to provide certain bit rate. For example, the transmission rate of 2 slots in frame and 1 code in each slot provides the same bit rate as that of 1 slot in frame and 2 codes in slot. Therefore, access point(AP) can provide various bit rates required by mobile terminals in many ways, depending on the status of available slots and spreading codes in system.

\section{B. System modeling}

The connection-oriented subsystem model of PCRMA is shown in Fig.2, where $\gamma_{c}$ and $o_{c}$ indicate the transition probabilities from state 'IDLE' to 'CON', and from 'RES $i, j$ ' to 'DLE', respectively. Mobile terminal which needs connection-oriented service enters 'CON' state, and transmits contention packet to the access point(AP) using available spreading codes which is randomly selected based on feedback message. Then, it waits until it receives feedback message from AP. Upon successful channel allocation, it goes to RES $i, j$ ' state, and remains in 'CON' state if contention fails. State 'RESi,j' 
indicates when mobile terminal is assigned with i-slots in frame, and j-spreading codes in each slot. When connection is released, mobile terminal goes to 'IDLE' state. Based on this model, we can represent PCRMA system state, and analyze the mean blocking probability and packet dropping probability based on equilibrium point analysis(EPA) method[2].

First, we can derive the transition probability from state 'CON' to RESi,j' of PCRMA by using the performance analysis method of [4]. Let $L$ denote the number of slots in frame, and let $N$ denote the number of spreading codes which can be used in each slot. Also, let $r_{c}$ and $r_{d}$ denote the permission probability of connectionoriented and connectionless terminals, respectively. Moreover, let $r$ denote the probability that certain spreading code is reserved, and let $l$ denote the number of available codes in a slot. In addition, let $c$ and $d$ denote the number of connection-oriented and connectionless mobile terminals in contention state, respectively. Then, a connection-oriented terminal in the 'CON' state gets a reservation on $\mathrm{j}$ spreading codes and enters into the 'RES $i, j$ ' state, as a result of the concurrence of the events $\left\{\boldsymbol{E}_{c k}, \kappa=U, 1, \Lambda, \mathcal{J}\right\}$ as described below:

$\boldsymbol{E}_{c 0}$ terminal does not enter a 'IDLE' state at the end of the slot

$\boldsymbol{E}_{c 1} J$ codes happen to be randomly selected by connection-oriented terminal from a pool of all available codes

$\boldsymbol{E}_{c 2}$ codes are unreserved

$\boldsymbol{E}_{c 3}$ terminal has permission $\left(\boldsymbol{r}_{c}\right)$ to transmit packets

$\boldsymbol{E}_{c 4}$. no other contending connection-oriented terminal selects these codes or gets permission to transmit a packet even if it selects these codes

$\boldsymbol{E}_{c 5}$ no contending connectionless terminal(with backlogged data packets) selects these codes, or obtains permission $\left(r_{d}\right)$ even if it selects these codes

Also, the probability of each event is given by Eq.1 Eq.6.

$$
\begin{aligned}
& \operatorname{Pr}\left\{E_{c 0}\right\}=\left(1-\gamma_{c}\right) \\
& \operatorname{Pr}\left\{E_{c 1}\right\}=\sum_{l=1}^{N}\left(\begin{array}{c}
N-j \\
l-j
\end{array}\right) r^{N-l}(1-r)^{l-j} \cdot \frac{j}{l} \\
& \operatorname{Pr}\left\{E_{c 2}\right\}=(1-r)^{j}
\end{aligned}
$$

$$
\begin{aligned}
& \operatorname{Pr}\left\{E_{c 3}\right\}=P_{c} \\
& \operatorname{Pr}\left\{E_{c 4}\right\}=\left(1-P_{c} \cdot \operatorname{Pr}\left\{E_{c 0}\right\}\right)^{c-1} \\
& \operatorname{Pr}\left\{E_{c 5}\right\}=\left(1-P_{d} \cdot \operatorname{Pr}\left\{E_{c 0}\right\}\right)^{d}
\end{aligned}
$$

Then, the probability that mobile terminal gets reservation on $i$ slots in frame and $j$ spreading codes in each slot, $J_{i, j}$, can be obtained as Eq.7.

In Eq.7, if $L=1(i=1)$ and $J=1$, then it becomes the same system as RCMA, as described in Eq.8 of ref[4]. Namely, if mobile terminal uses continuous slots $L=1$, $i=1)$ and single code in $\operatorname{siot}(y=1)$, then PCRMA becomes the same system as RCMA, where $J_{1,1}$ exists only. From these results, we can analyze the performance of PCRMA by using equilibrium point analysis(EPA) method[2]-[4].

Fig.3 shows the connectionless subsystem model, where the state means the number of packets in the transmission buffer of terminal which is waiting to be transmitted to the AP. Here, $w_{j}$ indicates the probability that terminal transmits $j$ packets using $j$ spreading codes in a certain slot. Then, $w_{j}$ is calculated as

$$
\begin{aligned}
w_{j}= & (1-r)^{j} \cdot P_{j}^{\prime} \cdot \\
& P_{d}\left(1-P_{c} \cdot P_{j}^{\prime}\right)^{c}\left(1-P_{d} \cdot P_{j}^{\prime}\right)^{d-1}
\end{aligned}
$$

\section{Performance analysis and numerical results A. Performance analysis}

Equilibrium point analysis(EPA) method is proposed by Tasaka[2] first, and used by Nanda[8] for the performance analysis of PRMA protocol. EPA method is simple and accurate in the sense that performance prediction of a stable system well agrees with the results using computer simulation, and is therefore adopted in our analysis. By definition, an equilibrium point is the point at which the expected increase in the number of users in each terminal state is zero. Let us denote system equilibrium state by $\omega$, and number of connectionoriented and connectionless terminals by $c, d$. Then, $\omega$ is represented as

$$
\omega=\left(s, c, r_{1}, r_{2}, r_{3}, \mathrm{~K}, r_{N}, b_{0}, b_{1}, \mathrm{~K}, b_{j}, \mathrm{~K}\right)
$$

Remember all the spreading codes are equally likely to be reserved. That is, $r_{1}=r_{2}=\Lambda=r_{N}=r$.

Refer to the subsystem models shown in Fig.2 and Fig.3. By assuming that entering flow is the same as

$$
\begin{gathered}
f_{i, j}=\left(1-\gamma_{c}\right) \cdot \frac{L !}{i !(L-i)} \cdot\left\{(1-r)^{j} \cdot P_{j}^{\prime}\right\} \cdot P_{c}\left(1-P_{c} \cdot P_{j}^{\prime}\right)^{-1} \cdot\left(1-P_{d} \cdot P_{j}^{\prime}\right)^{d}, 1 \leq i \leq L \text { and } 1 \leq j \leq N \\
\text { where } P_{j}^{\prime}=\sum_{l=1}^{N}\left(\begin{array}{c}
N-j \\
l-j
\end{array}\right) \gamma^{N-l}(1-r)^{l-j} \cdot \frac{j}{l} \\
c \gamma_{c}+N r \gamma_{c}=s \sigma_{c} \text { ("idle") } \\
r \gamma_{c}=c f_{c} \text { ("res") } \\
b_{0} \sigma_{d}=b_{1} w\left(1-\sigma_{d}\right)(\text { ("BUF") } \\
b_{0} \sigma_{d}+b_{2} w\left(1-\sigma_{d}\right)=b_{1} w\left(1-\sigma_{d}\right)+b_{1} \sigma_{d}(1-w)\left(" \mathrm{BUF}{ }_{1}\right. \text { ") } \\
b_{j}\left[w\left(1-\sigma_{d}\right)+\sigma_{d}(1-w)\right]=b_{j+1} w\left(1-\sigma_{d}\right)+b_{j-1} \sigma_{d}(1-w)\left(" \mathrm{BUF}_{j} ", j \geq 2\right)
\end{gathered}
$$


<Table.II> Design parameters of PCRMA protocol

\begin{tabular}{|l|c|c|l|}
\hline \multicolumn{1}{|c|}{ Definition } & notation & units & value \\
\hline frame duration & $I_{f}$ & $\mathrm{~ms}$ & 20.0 \\
\hline slots per frame & $K$ & & 20 \\
\hline slot width & $\tau$ & $\mathrm{ms}$ & 1.00 \\
\hline number of spreading codes available in a single slot & $N$ & & $40 / 120$ \\
\hline chip rate of spreading code & $R_{r}$ & $\mathrm{~kb} / \mathrm{s}$ & 1568 \\
\hline CDMA total bandwidth & $B_{w}$ & $\mathrm{MHz}$ & 1.25 \\
\hline connection duration of connection-oriented service & $L_{2}$ & $\mathrm{~s}$ & exponential \\
\hline source rate of connection-oriented service (voice) & $R_{c}$ & $\mathrm{~b} / \mathrm{s}$ & variable \\
\hline maximum allowable delay of connection-oriented service & $D_{c_{-}}$max & $\mathrm{ms}$ & 4 \\
\hline mean rate of connectionless service (data) & $R_{d}$ & $\mathrm{~b} / \mathrm{s}$ & 2400 \\
\hline mean delay time of connectionless service & $D_{a v}$ & $\mathrm{~ms}$ & variable \\
\hline number of total terminals & $M_{c}$ & & variable \\
\hline number of terminals using connection-oriented service & $M_{c}$ & & variable \\
\hline number of terminals using connectionless service & $M_{d}$ & & variable \\
\hline permission probability of connection-oriented service & $P_{c}$ & & variable \\
\hline permission probability of connectionless service & $P_{d}$ & & variable \\
\hline
\end{tabular}

leaving flow, Eq.10 Eq.14 can be driven.

From these equations, if the total numbers of connection-oriented and connectionless terminals is given, the system equilibrium state is characterized by parameters $b, c$ and $r$.

However, it is difficult to solve directly the above equations since equations are highly nonlinear. Therefore, we form equation of a single unknown symbol whose solution is relatively easy to obtain. Let us express above equations in terms of unknown $c$. Then, following equations can be obtained.

$$
\begin{gathered}
r=\frac{1}{N}\left(\frac{\sigma_{c}}{\sigma_{c}+r} M_{c}-c\right) \cong \psi_{r}(c) \\
w=\frac{N f_{c} P_{d}^{\prime}\left(1-r-P_{d}^{\prime}\right)}{P_{c}^{\prime}\left(1-\gamma_{c}\right)\left(1-r-P_{d}^{\prime}\right)} \\
b=\frac{M_{d} \sigma_{d R} c\left(1-\gamma_{c}\right)}{N r \gamma_{c}} \frac{P_{c}^{\prime}\left(1-r-P_{d}^{\prime}\right)}{P_{d}^{\prime}\left(1-r-P_{c}^{\prime}\right)}
\end{gathered}
$$

Eq.15 Eq.17 can be solved from unknown $c$. Then, we can derive the packet dropping and mean blocking probability of connection-oriented service, and mean delay of connectionless service.

Finally, in the case that mobile terminal in 'CON' state needs $i$ slots and $j$ spreading codes, the blocking probability $v$ is described as Eq.18. Also, in the case of interactive multimedia traffic, average packet dropping probability $\boldsymbol{\Gamma}_{\text {drop }}$ is calculated as Eq.19. In the case of connectionless service, mean packet delay is represented as Eq. 20.

\section{B. Numerical results}

In this section, we illustrate the performance of PCRMA protocol by applying it to a microcellular mobile radio network. Also, to show the relative performance, we compare the performance of PCRMA with that of reservation-code multiple-access(RCMA) [4] which is the CDMA wireless MAC protocol based on contention and reservation.

For performance comparison of PCRMA and RCMA, we use the mean blocking probability and packet dropping probability of terminals, considering connection-oriented multimedia services requiring various bit rates. We assume that neighboring microcells share frequency bandwidth using spreading-tone with chip rate of $\boldsymbol{K}_{c}=1568 \mathrm{~kb} / \mathrm{s}$, and total number of the spreading codes available in a slot $N$ is 40 or 120 . These system parameters for performance analysis is shown in Table II.

If we assume the channel rate of $32 \mathrm{Kbps}$, the maximum

$$
v=1-\gamma_{c}-\sum_{i=1}^{L} \sum_{j=1}^{N} f_{i, j}
$$

$$
\begin{gathered}
P_{d r o p}=\gamma_{c}\left[\frac{v \gamma_{c}}{\left[1-v\left(1-\gamma_{c}\right)\right]^{2}}+\frac{v^{D}\left(1-\gamma_{c}\right)^{D}}{1-v} \times\left\{1-\frac{v \gamma_{c}\left(2+v \gamma_{c}-2 v\right)}{\left[1-v\left(1-\gamma_{c}\right)\right]^{2}}\right\}\right] \\
D_{a v}=\frac{1-w}{w} \frac{1-\rho_{d} \sigma_{d}}{1-\rho_{d}} \quad \text { where, } \rho_{d}=\sigma_{d} / w
\end{gathered}
$$


bit rate of mobile terminal in RCMA becomes 32Kbps[4]. Therefore, we assume the maximum traffic source rate is the same as that of RCMA for fair comparison, though higher bit rate can be supported in the proposed PCRMA. Considering interactive voice and video traffic, the maximum queueing delay time is set to $100 \mathrm{~ms}$. For traffic source, we consider multimedia traffic sources requiring various bit rates such as $4800 \mathrm{bps}, 9600 \mathrm{bps}, 16 \mathrm{Kbps}$, and $32 \mathrm{Kbps}$. Also, the number of mobile terminals requiring each bit rate is assumed to be the same.

Fig.4 and Fig.5 show the mean blocking probability and average packet dropping probability versus the number of connection-oriented mobile terminals in the system. For fair comparison with RCMA, it is assumed that $r_{c}=r_{d}=1$ in PCRMA and slot width is $1 \mathrm{~ms}$, and $N=40$ and $N=120$ are considered. Also, considering interactive multimedia service, connection duration time of connection-oriented service is assumed to have exponential distribution with mean of $180 \mathrm{sec}$. Here, there are little difference between simulation and numerical results. This phenomenon occurs because we limited the maximum threshold in the random number generation in this simulation.

As shown in figures, PCRMA provides better

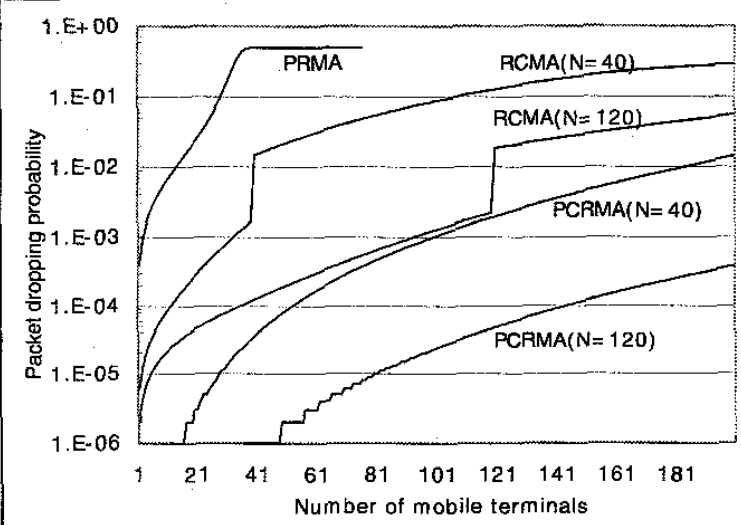

( $\mathrm{N}$ : number of spreading codes)

$<$ Fig.4> Mean packet dropping probability of connectionoriented service

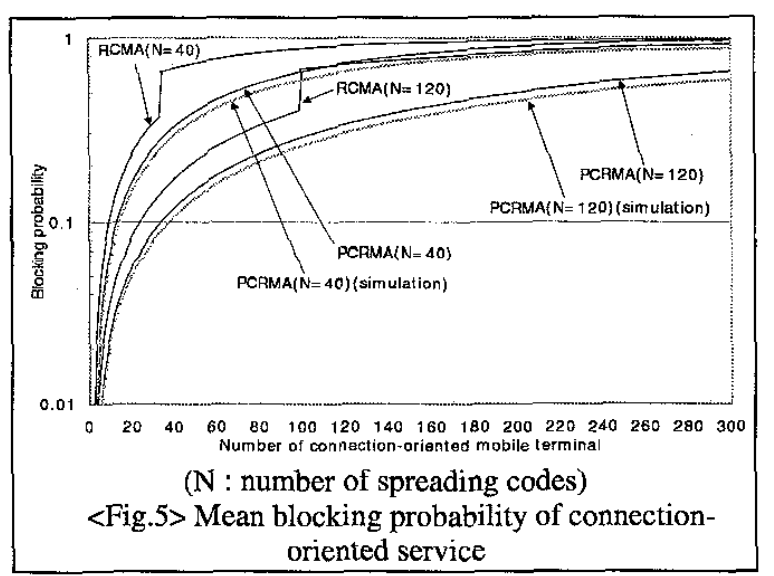

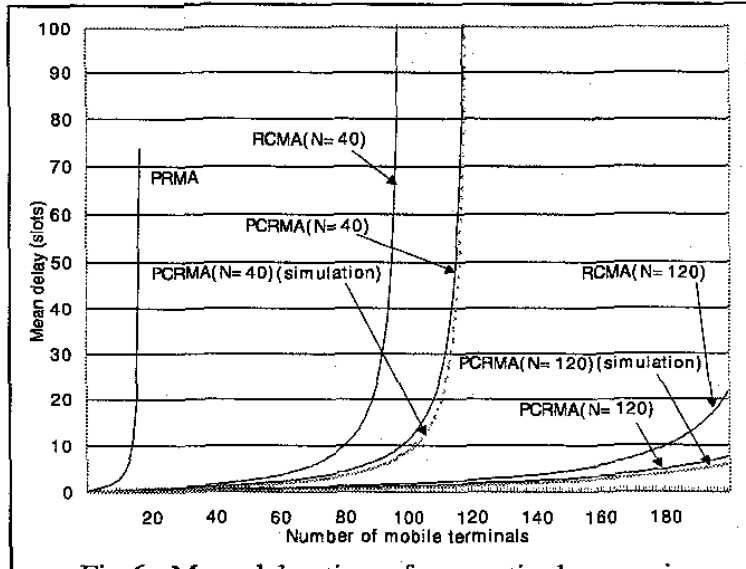

<Fig.6> Mean delay time of connectionless service

performance than RCMA, in terms of blocking probability and packet dropping probability. The reason is that non-continuous slots and multiple codes are assigned according to the required bit rate, and spreading code utilization efficiency is improved in PCRMA. However, in the case of RCMA, reserved single spreading code have to be used in continuous slots, and wireless channels are wasted in the case of traffic source with low bit rate. In addition, in PCRMA, mobile terminal can be provided with higher bit rate, unlike the RCMA. Thus, PCRMA network can provide integrated multimedia services with various bit rates, because of improvements of spreading code utilization efficiency.

The mean packet delay of connectionless service is shown in Fig.6, where the performance of PCRMA is better than that of RCMA. In PCRMA, the waiting time to transmit connectionless packet is reduced to slot length, and collision occurrence probability is lower than RCMA because mobile terminal randomly selects one of available spreading codes. In addition, since mobile terminal can transmit multiple packets using one or more available spreading codes in the same slot, PCRMA gives lower delay time compared with RCMA.

\section{Conclusions}

In this paper, we proposed and analyzed the performance of PCRMA protocol as a wireless MAC protocol for the CDMA network supporting various multimedia services. PCRMA is designed to make limited spreading codes shared among mobile terminals in the indoor and micro-cell CDMA network, and provides connection-oriented and connectionless services. Also, not only voice but also various multimedia traffics can be supported, and various bit rates according to traffic type can be supported. From numerical results, it is shown that PCRMA provides better performance in view of blocking probability and packet dropping probability, compared with RCMA which is contention and reservation-based wireless MAC protocol for CDMA network. Also, PCRMA can support higher bit rate to mobile terminals, unlike conventional systems. 


\section{References}

[1] W. C. Y. Lee, "Overview of cellular CDMA," IEEE Trans. Veh. Technol., vol.40, pp.291-302, 1991.

[2] S. Tasaka, "Stability and performance analysis of the R-ALOHA packet broadcast system," IEEE Trans. Computer, vol.32, no.8, pp.717-726, 1983.

[3] D. J. Goodman et al., "Packet reservation multiple access for local wireless communications," IEEE Trans. Commun., vol.37, no.8, pp.885-890, 1989.

[4] Lijun Tan and Qi Tu Zhang, "A Reservation RandomAccess Protocol for Voice/Data Integrated SpreadSpectrum Multiple-Access Systems", IEEE J. Selec. Areas Commun., vol.14, no.9, pp.1717-1727, 1996.

[5] M. J. McTiffin, A. P. Hulbert, T. J. Ketseoglou, W.
Heimsch, and G. Crisp, "Mobile Access to an ATM network using a CDMA air interface," IEEE J. Select. Areas Commun., vol.12, no.5, 1994.

[6] Shengming Jiang and Danny H. K. Tsang, "On Architecture for Broadband Wireless Systems," IEEE Comm. Mag., pp.132-140, October 1997.

[7] M. J. McTiffin, A. P. Hulbert, T. J. Ketseoglou, W. Heimsch, and G. Crisp, "Mobile Access To and ATM Network Using a CDMA Air Interface," IEEE J. Selec. Areas Commun., vol.12, no.5, Jun. 1993.

[8] S. Nanda, D. J. Goodman, and U. Timor, "Performance of PRMA: A packet voice protocol for cellular system," IEEE Trans. Veh. Technol., vol.40, no.3, pp.584-598, 1991.

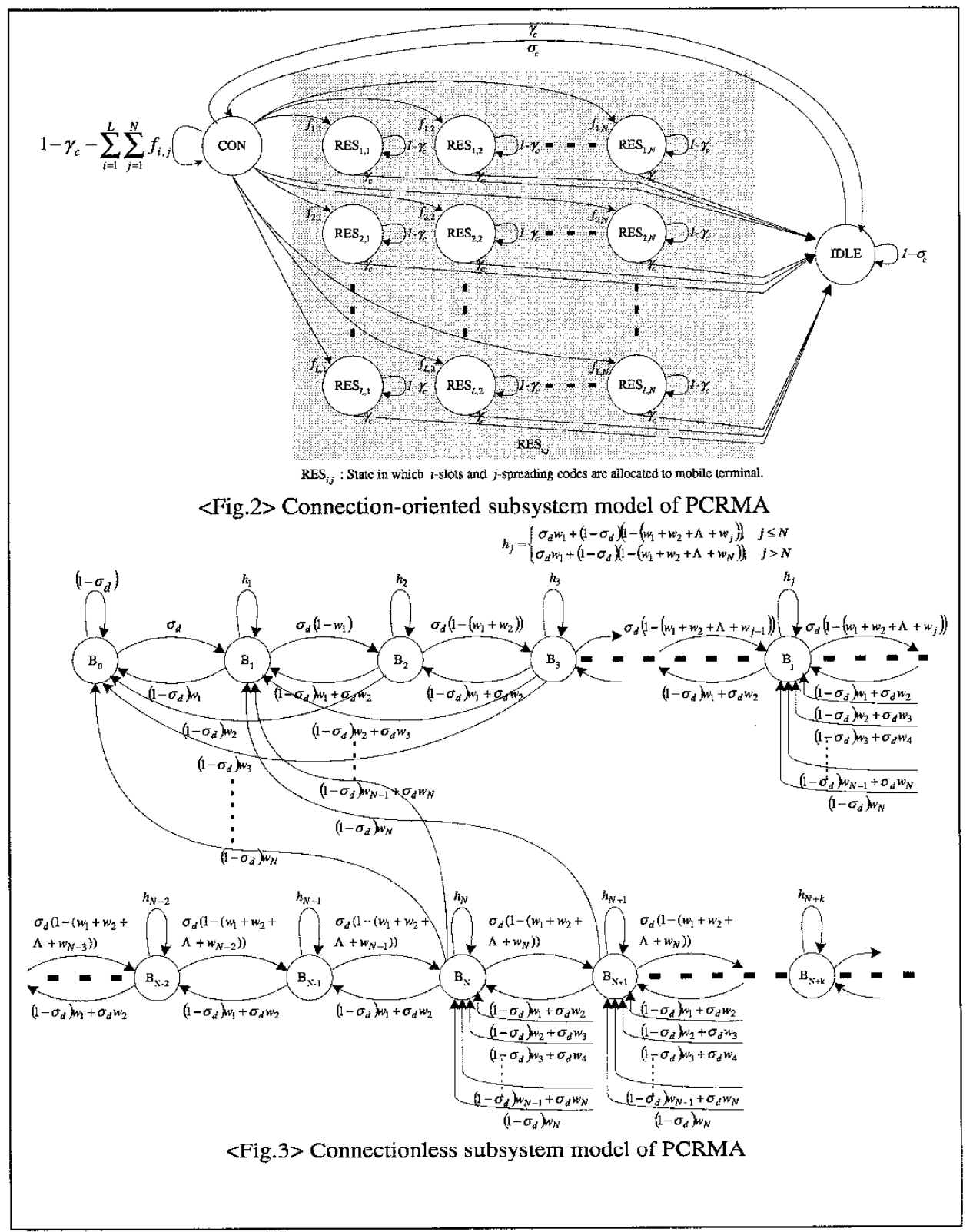

